\title{
Improved Measurement of Inclusive Radiative $B$-meson decays
}

K. Abe, I. Adachi, J. Brodzicka, J. Dragic, H. Fujii, J. Haba, M. Hazumi, T. Higuchi, Y. Igarashi, R. Itoh, Y. Iwasaki, N. Katayama, A. Kibayashi, H. Kichimi, P. Krokovny, J. MacNaughton, I. Nakamura, M. Nakao, S. Nishida, T. Nozaki, H. Ozaki, Y. Sakai, J. Schümann, K. Sumisawa, S. Y. Suzuki, O. Tajima, F. Takasaki, K. Tamai, M. Tanaka, K. Trabelsi, T. Tsuboyama, S. Uehara, S. Uno, Y. Ushiroda, and M. Yamauchi High Energy Accelerator Research Organization (KEK), Tsukuba

H. Aihara, N. C. Hastings, M. Iwasaki, H. Kakuno, A. Kusaka, Y. Nakahama, and H. Nakayama Department of Physics, University of Tokyo, Tokyo

K. Arinstein, V. Aulchenko, I. Bedny, A. Bondar, S. Eidelman, D. Epifanov, N. Gabyshev, A. Kuzmin, A. Poluektov, B. Shwartz, Y. Usov, A. Vinokurova, V. Zhilich, and V. Zhulanov Budker Institute of Nuclear Physics, Novosibirsk

T. Aso

Toyama National College of Maritime Technology, Toyama

T. Aushev

École Polytechnique Fédérale de Lausanne (EPFL), Lausanne and Institute for Theoretical and Experimental Physics, Moscow

T. Aziz, S. Banerjee, A. Das, G. Gokhroo, N. J. Joshi, and G. Majumder Tata Institute of Fundamental Research, Mumbai

S. Bahinipati, A. Drutskoy, P. Goldenzweig, K. Kinoshita, K. Sayeed, A. J. Schwartz, and A. Somov University of Cincinnati, Cincinnati, Ohio 45221

A. M. Bakich, S. Cole, S. McOnie, N. Parslow, L. S. Peak, K. E. Varvell, and B. D. Yabsley University of Sydney, Sydney, New South Wales

V. Balagura, R. Chistov, M. Danilov, D. Liventsev, T. Medvedeva, R. Mizuk, P. Pakhlov, G. Pakhlova, E. Solovieva, I. Tikhomirov, and T. Uglov Institute for Theoretical and Experimental Physics, Moscow

Y. Ban and J. Wang

Peking University, Beijing

E. Barberio, J. Dalseno, A. Limosani, G. R. Moloney, M. E. Sevior, G. N. Taylor, Y. F. Tse, P. Urquijo, and R. Wedd University of Melbourne, School of Physics, Victoria 3010

A. Bay, L. Hinz, C. Jacoby, T. Schietinger, O. Schneider, K. Vervink, S. Villa, J. Wicht, and N. Zwahlen École Polytechnique Fédérale de Lausanne (EPFL), Lausanne

K. Belous, M. Shapkin, and A. Sokolov Institute of High Energy Physics, Protvino

V. Bhardwaj, R. Kumar, and J. B. Singh Panjab University, Chandigarh

U. Bitenc, S. Fratina, R. Pestotnik, M. Starič, and A. Zupanc J. Stefan Institute, Ljubljana

S. Blyth and C. H. Wang National United University, Miao Li

A. Bozek, P. Kapusta, T. Lesiak, A. Matyja, Z. Natkaniec, W. Ostrowicz, H. Palka, M. Rozanska, J. Stypula, and J. Wiechczynski 
H. Niewodniczanski Institute of Nuclear Physics, Krakow

$$
\begin{gathered}
\text { M. Bračko and S. Korpar } \\
\text { University of Maribor, Maribor and } \\
\text { J. Stefan Institute, Ljubljana }
\end{gathered}
$$

T. E. Browder, H. Guler, H. Hoedlmoser, J. Li, K. Nishimura, S. L. Olsen, M. Peters, J. Rorie, H. Sahoo, K. Uchida, and G. Varner University of Hawaii, Honolulu, Hawaii 96822

$$
\begin{gathered}
\text { M.-C. Chang } \\
\text { Department of Physics, Fu Jen Catholic University, Taipei }
\end{gathered}
$$

P. Chang, Y. Chao, K.-F. Chen, C.-C. Chiang, W.-S. Hou, Y. B. Hsiung, S.-W. Lin, J.-G. Shiu, K. Ueno, C. C. Wang, and M.-Z. Wang Department of Physics, National Taiwan University, Taipei

A. Chen, W. T. Chen, A. Go, S. Hou, and H. Nakazawa National Central University, Chung-li

B. G. Cheon and Y. Unno Hanyang University, Seoul

I.-S. Cho, J. H. Kang, and Y.-J. Kwon Yonsei University, Seoul

S.-K. Choi

Gyeongsang National University, Chinju

Y. Choi, Y. K. Choi, H. O. Kim, J. H. Kim, J. S. Lee, C. W. Park, and K. S. Park Sungkyunkwan University, Suwon

M. Dash, L. E. Piilonen, and Y. Yusa

Virginia Polytechnic Institute and State University, Blacksburg, Virginia 24061

M. Fujikawa, H. Hayashii, S. U. Kataoka, K. Miyabayashi, S. Noguchi, and A. Sekiya Nara Women's University, Nara

\author{
A. Garmash and D. Marlow \\ Princeton University, Princeton, New Jersey 08544 \\ B. Golob and P. Križan \\ University of Ljubljana, Ljubljana and \\ J. Stefan Institute, Ljubljana \\ M. Grosse Perdekamp and R. Seidl \\ University of Illinois at Urbana-Champaign, Urbana, Illinois 61801 and \\ RIKEN BNL Research Center, Upton, New York 11973 \\ H. Ha and E. Won \\ Korea University, Seoul
}

K. Hara, K. Hayasaka, T. Hokuue, T. Iijima, K. Ikado, K. Inami, M. Kaga, H. Kaji, Y. Kozakai, T. Matsumura, Y. Miyazaki, T. Mori, Y. Nishio, T. Ohshima, K. Senyo, and M. Yamaoka Nagoya University, Nagoya

T. Hara, D. Heffernan, S. Kajiwara, H. Miyake, and S. Shinomiya

Osaka University, Osaka 
Y. Hasegawa and N. Satoyama

Shinshu University, Nagano

Y. Horii, Y. Mikami, T. Nagamine, P. Schönmeier, A. Yamaguchi, and H. Yamamoto Tohoku University, Sendai

Y. Hoshi and K. Neichi

Tohoku Gakuin University, Tagajo

K. Hoshina and O. Nitoh

Tokyo University of Agriculture and Technology, Tokyo

H. J. Hyun, D. H. Kah, H. J. Kim, and H. Park

Kyungpook National University, Taegu

A. Ishikawa, A. Murakami, A. Sugiyama, and S. Suzuki Saga University, Saga

H. Ishino and S. Ono

Tokyo Institute of Technology, Tokyo

M. Iwabuchi, Y. J. Kim, Y. Liu, T. R. Sarangi, and Y. Uchida

The Graduate University for Advanced Studies, Hayama

H. Kawai and E. Kurihara

Chiba University, Chiba

T. Kawasaki, H. Miyata, N. Tamura, and M. Watanabe Niigata University, Niigata

S. K. Kim, J. Lee, M. J. Lee, and S. E. Lee Seoul National University, Seoul

J. S. Lange

Justus-Liebig-Universität Gießen, Gießen

G. Leder, F. Mandl, W. Mitaroff, M. Pernicka, C. Schwanda, and L. Widhalm Institute of High Energy Physics, Vienna

J. Mueller

University of Pittsburgh, Pittsburgh, Pennsylvania 15260

Y. Nagasaka

Hiroshima Institute of Technology, Hiroshima

E. Nakano, H. Sakaue, and Y. Teramoto Osaka City University, Osaka

I. Nishizawa and T. Sumiyoshi

Tokyo Metropolitan University, Tokyo

A. Ogawa

RIKEN BNL Research Center, Upton, New York 11973

S. Ogawa and H. Shibuya

Toho University, Funabashi

S. Okuno and Y. Watanabe 
Kanagawa University, Yokohama

N. Sasao and N. Taniguchi

Kyoto University, Kyoto

L. Shang, C. P. Shen, P. Wang, X. L. Wang, C. Z. Yuan, and C. C. Zhang

Institute of High Energy Physics, Chinese Academy of Sciences, Beijing

\author{
S. Stanič \\ University of Nova Gorica, Nova Gorica \\ Y. Yamashita \\ Nippon Dental University, Niigata \\ L. M. Zhang and Z. P. Zhang \\ University of Science and Technology of China, Hefei
}

\begin{abstract}
We report a fully inclusive measurement of the flavor changing neutral current decay $B \rightarrow X_{s} \gamma$ in the energy range $1.7 \mathrm{GeV} \leq E_{\gamma}^{\text {c.m.s }} \leq 2.8 \mathrm{GeV}$, covering $97 \%$ of the total spectrum, where c.m.s is the center of mass system. Using $605 \mathrm{fb}^{-1}$ of data, we obtain in the rest frame of the $B$-meson $\mathcal{B}\left(B \rightarrow X_{s} \gamma: E_{\gamma}^{B}>1.7 \mathrm{GeV}\right)=(3.31 \pm 0.19 \pm 0.37 \pm 0.01) \times 10^{-4}$, where the errors are statistical, systematic and from the boost correction needed to transform from the rest frame of the $\Upsilon(4 S)$ (c.m.s) to that of the $B$-meson, respectively. We also measure the first and second moments of the photon energy spectrum as functions of various energy thresholds, which extend down to $1.7 \mathrm{GeV}$. The results are preliminary.
\end{abstract}

PACS numbers: $12.39 . \mathrm{Hg}, 13.20 . \mathrm{He}, 13.40 . \mathrm{Hq}, 14.40 . \mathrm{Nd}$, 14.65.Fy

Radiative $B$-meson decays may offer a view of phenomena beyond the Standard Model of particle physics (SM). In the SM, these decays proceed via a flavor changing neutral current (FCNC) decay, which consists of a loop process. Yet to be discovered particles, such as charged Higgs or supersymmetric particles, may be produced virtually in the loop and produce a measureable deviation from the branching fraction predicted by the SM.

The predictions of the branching fraction at order $\alpha_{s}^{2}$ $(\mathrm{NNLO}-$ next to next to leading order) $(3.15 \pm 0.23) \times$ $10^{-4}$ [1], $(2.98 \pm 0.26) \times 10^{-4}$ [2] and the average of experiment measured values $(3.55 \pm 0.26) \times 10^{-4}[3]$ are in tacit agreement. An updated experimental measurement would further test this agreement, and, moreover, give stronger constraints on extensions to the SM e.g. Minimal Supersymmetric Standard Model [4] and left-right symmetric models [5, [6] .

The photon energy spectrum is also of great importance. At the parton level, the photon is monochromatic with energy $E \approx m_{b} / 2$ in the $b$-quark rest frame. The energy is smeared by the motion of the $b$-quark inside the $B$ meson and gluon emission. A measurement of the moments of this spectrum allows for a determination of the $b$-quark mass and of its Fermi motion. This information can then be used in the extraction of the CKM matrix elements $\left|V_{c b}\right|$ and $\left|V_{u b}\right|$ from inclusive semileptonic $B$ decays [7]. A measurement of the low-energy tail of the photon spectrum is important in this context [8].

Belle has previously measured the $B \rightarrow X_{s} \gamma$ branching fraction with $5.8 \mathrm{fb}^{-1}$ and $140 \mathrm{fb}^{-1}$ of data using semi-inclusive [9] and fully inclusive approaches [10], respectively. Other measurements include those from CLEO [11] and BaBar [12, 13, 14].

Here we present an update of our fully inclusive measurement [10], based on a much larger dataset and with significant refinements, which includes an unfolding of detector effects on the measured spectrum that improve the measurements of the branching fraction and spectral moments, respectively. We also extend the photon energy range to $E_{\gamma}^{\text {c.m.s }}>1.7 \mathrm{GeV}$, covering more of the spectrum than ever before, where c.m.s refers to the centre of mass system, which is equivalent to the rest frame of the $\Upsilon(4 S)$.

The $B \rightarrow X_{s} \gamma$ decay is studied using the Belle detector at the KEKB asymmetric $e^{+} e^{-}$storage ring [15]. The data consists a of sample of $604.6 \mathrm{fb}^{-1}$ taken at the $\Upsilon(4 S)$ resonance corresponding to $(656.7 \pm 8.9) \times 10^{6} B \bar{B}$ pairs. Another $68.3 \mathrm{fb}^{-1}$ sample has been taken at an energy $60 \mathrm{MeV}$ below the resonance and is used to measure the non- $B \bar{B}$ background. Throughout this manuscript, we refer to these data samples as the ON and OFF samples, respectively.

The Belle detector is a large-solid-angle magnetic spectrometer described in detail elsewhere [16]. The main component relevant for this analysis is the electromagnetic calorimeter (ECL) made of 16.2 radiation lengths long $\mathrm{CsI}(\mathrm{Tl})$ crystals. The photon energy resolution is about $2 \%$ for the energy range relevant in this analysis.

The strategy to extract the signal $B \rightarrow X_{s} \gamma$ spectrum is to collect all high-energy photons, vetoing those originating from $\pi^{0}$ and $\eta$ decays to two photons. The contribution from continuum $e^{+} e^{-} \rightarrow q \bar{q}(q=u, d, s, c)$ and 
QED type events is subtracted using the OFF sample. The remaining backgrounds from $B \bar{B}$ events are subtracted using Monte Carlo (MC) distributions scaled by data control samples.

Photon candidates are selected from ECL clusters of $5 \times 5$ crystals in the barrel region $(-0.35 \leq \cos \theta \leq 0.70$, where $\theta$ is the polar angle with respect to the beam axis, subtended from the direction opposite the positron beam. They are required to have an energy $E_{\gamma}^{\text {c.m.s }}$ larger than $1.4 \mathrm{GeV}$. We require $95 \%$ of the energy to be deposited in the central $3 \times 3$ crystal array and use isolation cuts to veto photons from bremsstrahlung and interaction with matter. The center of the cluster has to be displaced from any other ECL cluster with $E>20 \mathrm{MeV}$ by at least $30 \mathrm{~cm}$ at the surface of the calorimeter, and from any reconstructed track by $30 \mathrm{~cm}$, or by $50 \mathrm{~cm}$ for tracks with a measured momentum above $1 \mathrm{GeV} / c$. Moreover, the angle between the photon and the highest energy lepton in the event has to be larger than 0.3 radians at the interaction point.

In the Belle detector, a non-negligible background (1\%) is due to the overlap of a hadronic event with energy deposits left in the calorimeter by previous QED interactions (mainly Bhabha scattering). Such composite events are completely removed using timing information for calorimeter clusters associated with the candidate photons. The cluster timing information is stored in the raw data, and is available in the reduced format used for analysis only for data processed after the summer of 2004. This divides our data set into $253.7 \mathrm{fb}^{-1}$ and $350.9 \mathrm{fb}^{-1}$ samples of reprocessed data without and with timing information, respectively. To minimise composite background due to Bhabha scattering and two-photon processes that contaminate both $\Upsilon(4 S)$ and continuum data samples, we veto any candidate that contains an ECL cluster with energy exceeding $1 \mathrm{GeV}$ within a cone of 0.2 radians in the direction opposite our photon candidate as measured in the c.m.s frame. In the second data set only photons that are in time with the rest of the event are retained. The efficiency of this selection on signal events is larger than $99.5 \%$. We veto candidate photons from $\pi^{0}$ and $\eta$ decays to two photons by combining each $B \rightarrow X_{s} \gamma$ candidate photon with all other photons in the event. We reject the photon candidate if the likelihood of being a $\pi^{0}$ or $\eta$ is larger than 0.1 and 0.2 , respectively, these yield, on average, background suppression factors of 4 and 2, respectively. These likelihoods are determined from MC and are functions of the laboratory energy of the other photon, its polar angle $\theta$ and the mass of the two-photon system.

In order to reduce the contribution from continuum events, we use two Fisher discriminants calculated in the c.m.s frame. The first discriminant exploits the topology of $B \rightarrow X_{s} \gamma$ events and combines three energy flows around the photon axis. These energy flow variables are obtained using all particles, except for the photon candidate, we measure the energy in the three regions defined by $\Theta<30^{\circ}, 30^{\circ} \leq \Theta \leq 140^{\circ}, \Theta>140^{\circ}$, where $\Theta$ is the angle of the particle to the candidate photon. The second exploits the spherical shape of $B \bar{B}$ events and is built using ten event-shape variables including Fox-Wolfram moments [17] for the full event and for the partial event with the photon removed, the full- and partial-event thrusts and the angles of the thrust axis with respect to the beam and the photon direction. To optimise these selection criteria, we use a MC simulation [18] containing large samples of $B \bar{B}, q \bar{q}$ and signal weighted according to the luminosities of the $\mathrm{ON}$ and OFF samples. In the optimisation step the signal MC used is generated as inclusive $B \rightarrow X_{s} \gamma$ and exclusive $B \rightarrow K^{*} \gamma$. The inclusive component $X_{s}$ is defined as a resonance of spin-1 with a Breit-Wigner form and a mass of $2.4 \mathrm{GeV} / c^{2}$ and width $1.5 \mathrm{GeV} / c^{2}$. The $X_{s}$ system is hadronised by JETSET and subsequently reweighted to match the prediction of the DGE model [19] 34] with $m_{b}(\overline{\mathrm{MS}})=4.20 \mathrm{GeV} / c^{2}$, with the mass extending no lower than $1.18 \mathrm{GeV} / c^{2}$ to agree with the corresponding world average branching fractions [3]. To improve the understanding of the photon energy spectrum at low energies, the selection criteria are optimised to maximize the sensitivity to the signal in the energy bin $1.8 \mathrm{GeV}<E_{\gamma}^{\text {c.m.s }}<1.9 \mathrm{GeV}$.

After these selection criteria we observe $4.15 \times 10^{6}$ and $0.25 \times 10^{6}$ photon candidates in the $\mathrm{ON}$ and $\mathrm{OFF}$ data samples, respectively. The spectrum measured in OFF data is scaled by luminosity to the expected number of non- $B \bar{B}$ events in ON data and subtracted. The formula used to subtract continuum background is as follows:

$$
\begin{aligned}
N^{B \bar{B}}\left(E_{\gamma}^{\text {c.m.s }(\mathrm{ON})}\right)= & N^{\mathrm{ON}}\left(E_{\gamma}^{\text {c.m.s }(\mathrm{ON})}\right)- \\
& \alpha \cdot \frac{\epsilon_{\text {Hadronic }}^{\mathrm{ON}}}{\epsilon_{\text {Hadronic }}^{\text {OFF }}} \cdot \frac{\epsilon_{B \rightarrow X_{s} \gamma}^{\mathrm{ON}}}{\epsilon_{B \rightarrow X_{s} \gamma}^{\mathrm{OFF}}} \cdot F_{N} \\
& \cdot N^{\mathrm{OFF}}\left(F_{E} E_{\gamma}^{\text {c.m.s }(\mathrm{OFF})}\right)
\end{aligned}
$$

where $\epsilon$ is the efficiency of Belle's hadronic selection 20] or of this analysis' $\left(B \rightarrow X_{s} \gamma\right)$ selection criteria in continuum events at either ON resonance $(\sqrt{s}=10.58 \mathrm{GeV})$ or OFF resonance $(\sqrt{s}=10.52 \mathrm{GeV})$ energies, and $\alpha$ is the ratio of ON to OFF resonance integrated luminosity corrected for the energy difference $(\alpha=8.7557( \pm 0.3 \%))$. The factors $F_{E}$ and $F_{N}$ compensate for the slightly lower mean energy and multiplicity of particles in OFF compared to $\mathrm{ON}$ events. We find $F_{N}=1.0009 \pm 0.0001$, $F_{E}=1.0036 \pm 0.0001, \frac{\epsilon_{\mathrm{Hadronic}}^{\mathrm{ON}}}{\epsilon_{\mathrm{Hadronic}}^{\mathrm{OF}}}=0.9986 \pm 0.0001$, and $\frac{\epsilon_{B \rightarrow X_{s} \gamma}^{\mathrm{ON}}}{\epsilon_{B \rightarrow X_{s} \gamma}^{\mathrm{OPF}}}=0.9871 \pm 0.0014$. The $\mathrm{ON}$ and scaled OFF spectra and their difference are shown in Fig. 1.

We then subtract the backgrounds from $B$ decays from the obtained spectrum. Six background categories are considered: (i) photons from $\pi^{0} \rightarrow \gamma \gamma$; (ii) photons from $\eta \rightarrow \gamma \gamma$; (iii) other real photons (mainly decays of $\omega, \eta^{\prime}$, and $J / \psi$, and bremsstrahlung, including the short distance radiative correction (modelled with PHOTOS 21]); (iv) ECL clusters not due to single photons (mainly $K_{L}^{0}$ 's and $\bar{n}$ 's); (v) Electrons misidentified as photons and; (vi) 


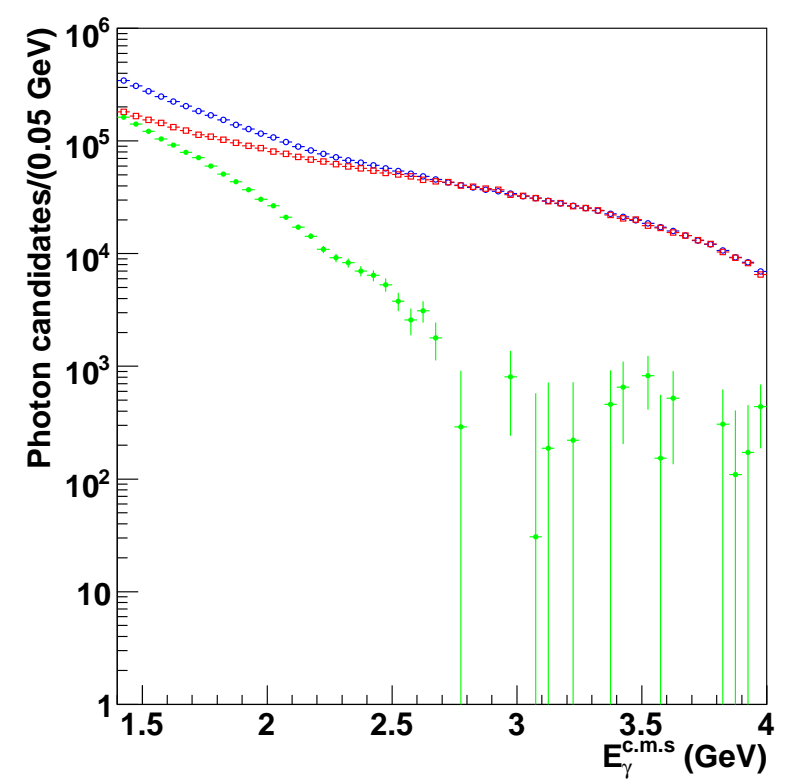

FIG. 1: ON data (open circle), scaled OFF data (open square) and continuum background subtracted (filled circle) photon energy spectra of candidates in the c.m.s frame.

\begin{tabular}{|l|c|}
\hline Contribution & Fraction \\
\hline Signal & 0.190 \\
\hline Decays of $\pi^{0}$ & 0.474 \\
Decays of $\eta$ & 0.163 \\
Other secondary $\gamma$ & 0.081 \\
Mis-IDed electrons & 0.061 \\
Mis-IDed hadrons & 0.017 \\
Beam background & 0.013 \\
\hline
\end{tabular}

TABLE I: Relative contributions of the $B \bar{B}$ backgrounds after selection in the $1.7<E_{\gamma}^{\text {c.m.s }} /(\mathrm{GeV})<2.8$ range

beam background. The spectra of the background of photons from $B$-meson decays with respect to the expected signal is shown in Fig. 2 and listed in Table [1 The net background of this type is a factor five greater than the signal.

For each of these categories we take the predicted background from $\mathrm{MC}$ and scale it according to measured yields wherever possible. The inclusive $B \rightarrow \pi^{0} X$ and $B \rightarrow \eta X$ spectra are measured in data using pairs of photons with well-balanced energies and applying the same $\mathrm{ON}-\mathrm{OFF}$ subtraction procedure. The yields obtained in data are on average $10 \%$ larger and $5 \%$ lower for $\pi^{0}$ and $\eta$ than MC expectations. The observed discrepancy between the measured and simulated $\pi^{0} \eta$ spectra is attributed to the branching fraction assumptions used for the generator 22]. Beam background is measured using a sample of randomly triggered events and added to the $B \bar{B}$ MC.

For each selection criterion and each background category we determine the $E_{\gamma}^{\text {c.m.s }}$-dependent selection effi-

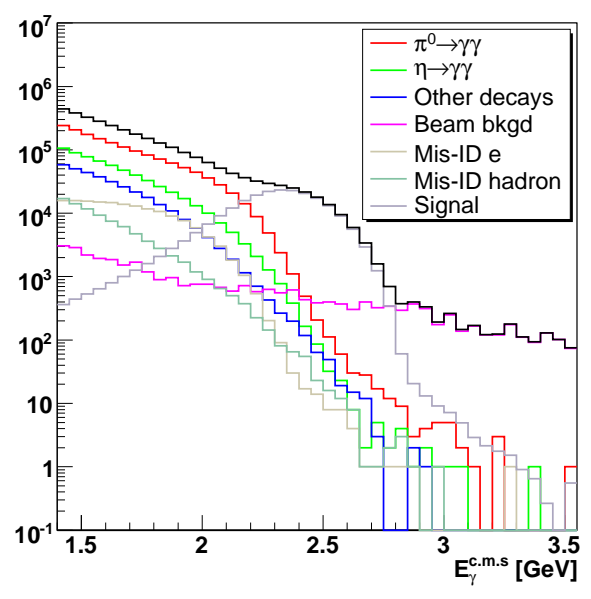

FIG. 2: The spectra of photons from $B$-meson decays passing selection criteria as predicted using a MC sample.

ciency in OFF-subtracted ON data and MC using appropriate control samples. We then scale the MC background sample according to the ratio of these efficiencies. The efficiencies of the $\pi^{0}$ and $\eta$ vetoes for photons not from $\pi^{0}$ and $\eta$ are measured in data using one photon from a reconstructed $\pi^{0}$, where the other photon of the $\pi^{0}$ is excluded from the search over the remaining photons for the next best $\pi^{0}$ or $\eta$ candidate (highest $\pi^{0}$ or $\eta$ likelihood). Consequently the best formed $\pi^{0}$ or $\eta$ candidate used in the calculation of the likelihoods is most likely a random combination, and therefore suited to measuring the effect of the vetoes. The $\pi^{0}$ veto efficiency is measured using a sample of photons coming from measured $\pi^{0}$ decays. We use partially reconstructed $D^{*+} \rightarrow D^{0} \pi^{+}$, $D^{0} \rightarrow K^{-} \pi^{+} \pi^{0}$ decays where the $\pi^{0}$ is replaced by the candidate photon in the reconstruction. The $\eta$ veto efficiency for photons from $\pi^{0}$ 's and event-shape criteria efficiencies are measured using a $\pi^{0}$ anti-veto sample, which is made of photons with a $\pi^{0}$ likelihood larger than 0.75 (i.e, no $\pi^{0}$ veto) and passing all other selection criteria. Other efficiencies are measured using the signal sample. Beam background is negligible after the application of the OFF time veto. In the sample of data where the veto is unavailable we scale the background according to a comparison of yields between MC and data for high energy $\left(E_{\gamma}^{\text {c.m.s }}>2.8 \mathrm{GeV}\right)$ photon candidates found in the endcaps of the ECL. This sample after continuum subtraction is a clean sample of ECL clusters from beam backgrounds.

The ratios of data and MC efficiencies versus $E_{\gamma}^{\text {c.m.s }}$ are fitted using first or second order polynomials, which are used to scale the background MC. Most are found to be statistically compatible with unity. An example is the effect of the $\pi^{0}$ veto on photons from $\pi^{0} \mathrm{~s}$ that escape the veto in the partially reconstructed $D^{*}$ sample, which is shown in Fig. 3 .

An exception is the efficiency of the requirement that 

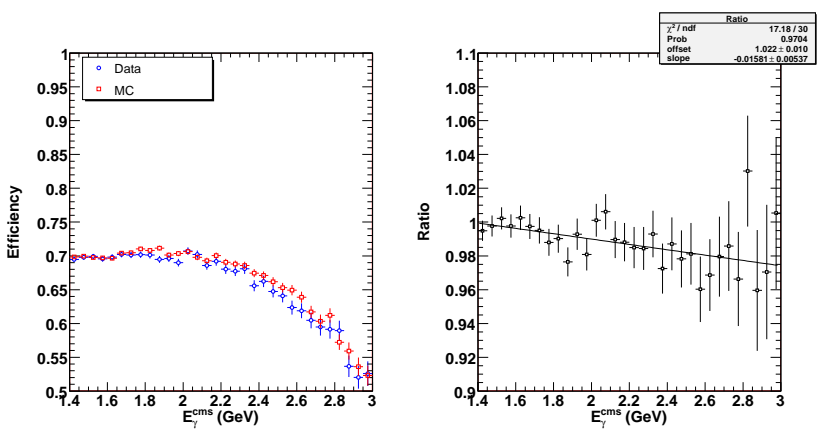

FIG. 3: (LEFT) The $\pi^{0}$ veto efficiency in the partially reconstructed $D^{*}$ sample for both Data (circles) and MC (squares) and (RIGHT) their ratio fitted with a first order polynomial.

$95 \%$ of the energy be deposited in the central nine cells of the $5 \times 5$ cluster, which is found to be poorly modelled by our MC for non-photon backgrounds. We estimate the efficiency for data using a sample of candidate photons in OFF-subtracted ON data after subtracting the known contribution from real photons. This increases the yield of background (iv) by $50 \%$. The yield from the six background categories, after having been properly scaled by the above described procedures, are subtracted from the OFF-subtracted spectrum. The result is shown in Fig. 4. After these subtractions the yield in the spectrum above the endpoint of $B$ decays is compatible with zero, $1245 \pm 4349$ candidates.

To measure the branching fraction and the moments we correct the raw spectrum using a three step procedure: (i) divide by the efficiency of the selection criteria i.e. the probability of a photon candidate passing cuts given a cluster has been found in the ECL, as a function of the measured energy in the c.m.s frame; (ii) perform an unfolding procedure based on the Singular Value Decomposition (SVD) algorithm [23], which maps the spectrum from measured energy to true energy thereby undoing the distortion caused by the ECL; (iii) divide by the efficiency of detection i.e. the probability that a photon originating at the interaction point is reconstructed in the ECL, as a function of the true energy. Data are divided into $50 \mathrm{MeV}$ wide bins. Step (ii), which was not performed in our previous analysis, is essential for a consistent extraction of partial branching fractions and moments as a function of lower energy thresholds. The unfolding matrix, derived from signal MC, is calibrated to data using the results of a study of radiative di-muon events, which gave the ECL response in data and MC in an energy and acceptance range consistent with our analysis. We use five signal models: KN [24], BLNP [25, 26], DGE [27], BBU [28] and GG [29]. Values of the parameters of the signal model used in the signal MC are derived from fits to the signal spectrum shown in Fig 4 . The two error bars for each point show the statistical and the total error, including the systematic error which is correlated among the points. In order to obtain the total $B \rightarrow X_{s} \gamma$ branching fraction we apply corrections for the contribution from Cabibbo suppressed $B \rightarrow X_{d} \gamma$ decays. The ratio of the $B \rightarrow X_{s} \gamma$ and $B \rightarrow X_{d} \gamma$ branching fractions is assumed to be $R_{d / s}=(4.0 \pm 0.4) \%$ [30]. We apply corrections to derive the measurements in the $B$ meson rest frame, using a toy $\mathrm{MC}$ approach. We generate photon 4-momentum in the rest frame of the $B$-meson using signal models referred to earlier, and generate $B$ meson 4-momentum using their known fixed energy and $1-\cos \theta^{2}$ distribution in the c.m.s. Repeating this exercise many times yields photon energy spectra in the rest frame of the $B$-meson and the c.m.s, from which we extract corrections used to yield measurements in the $B$ meson frame. The correction is derived as a mean over all signal models while the root-mean-square is assigned as the uncertainty. After correcting for the acceptance we derive distributions of the partial branching fraction, first moment (mean) and second central moment (variance) of $B \rightarrow X_{s} \gamma$ as measured in the c.m.s and $B$ rest frame for lower energy thresholds as shown in Fig. 囵 In the range from 1.7 to $2.8 \mathrm{GeV}$ in the rest frame of the $B$ meson, we obtain a partial branching fraction, and the first two moments of the energy spectrum:

$$
\begin{gathered}
\mathcal{B}\left(B \rightarrow X_{s} \gamma\right)=(3.31 \pm 0.19 \pm 0.37 \pm 0.01) \times 10^{-4} \\
\left\langle E_{\gamma}\right\rangle=2.281 \pm 0.032 \pm 0.053 \pm 0.002 \mathrm{GeV} \\
\left\langle E_{\gamma}^{2}\right\rangle-\left\langle E_{\gamma}\right\rangle^{2}=0.0396 \pm 0.0156 \pm 0.0214 \pm 0.0012 \mathrm{GeV}^{2},
\end{gathered}
$$

where the errors are statistical, systematic and from the boost correction, respectively.

The full results, the systematic error budget and correlation coefficients for five lower energy thresholds $\left(E_{\gamma}^{B}=\right.$ 1.7, 1.8, 1.9, 2.0, $2.1 \mathrm{GeV}$ ) are listed in Table $\amalg$. The total systematic error is derived from a sum in quadrature over all sources. We vary the number of $B \bar{B}$, the $\mathrm{ON}$ to OFF ratio of integrated luminosities and the correction factors applied to the OFF data photon candidates and assign the observed variation as the systematic associated with continuum subtraction. The parameters of the correction functions applied to the $\pi^{0}$ and $\eta$ yields are varied taking into account their correlations. As we do not measure the yields of photons from sources other than $\pi^{0}$ 's and $\eta$ 's in $B \bar{B}$ events, we independently vary the expected yields of these additional sources by $\pm 20 \%$. For the model dependence in correcting for the acceptance we use four signal models in addition to the default model, and assign the maximum deviation from the default as the uncertainty. The error on the photon detection efficiency in the ECL is measured to be $2 \%$ using radiative $\mu$-pair events, and also affects the estimation of photons from $B \bar{B}$. For the uncertainties related to the unfolding procedure, we vary the effective rank parameter up and down by one in the SVD algorithm.

In conclusion, we have measured the branching fraction and photon energy spectrum of $B \rightarrow X_{s} \gamma$ in the 
TABLE II: The measurements and correlation coefficients of the branching fraction, mean and variance of the photon energy spectrum for various lower energy thresolds, $E_{\gamma}^{B}$, as measured in the rest frame of the $B$-meson and the contributions to the systematic uncertainty.

\begin{tabular}{|c|c|c|c|c|c|c|c|c|c|c|c|c|c|c|c|}
\hline & \multicolumn{5}{|c|}{$\mathcal{B}\left(B \rightarrow X_{s} \gamma\right)\left(10^{-4}\right)$} & \multicolumn{5}{|c|}{$\left\langle E_{\gamma}\right\rangle(\mathrm{GeV})$} & \multicolumn{5}{|c|}{$\Delta E_{\gamma}^{2} \equiv\left\langle E_{\gamma}^{2}\right\rangle-\left\langle E_{\gamma}\right\rangle^{2}\left(\mathrm{GeV}^{2}\right)$} \\
\hline$E_{\gamma}^{\mathrm{B}}[\mathrm{GeV}]$ & 1.7 & 1.8 & 1.9 & 2.0 & 2.1 & 1.7 & 1.8 & 1.9 & 2.0 & 2.1 & 1.7 & 1.8 & 1.9 & 2.0 & 2.1 \\
\hline Value & 3.31 & 3.24 & 3.12 & 2.94 & 2.62 & 2.281 & 2.290 & 2.305 & 2.326 & 2.350 & $\overline{0.0396}$ & 0.0350 & 0.0292 & 0.0227 & 0.0170 \\
\hline \pm statistical & 0.19 & 0.17 & 0.15 & 0.14 & 0.12 & 0.032 & 0.025 & 0.019 & 0.015 & 0.011 & 0156 & 0.0096 & 0.0058 & 0.0033 & 0.0017 \\
\hline \pm systematic & 0.37 & 0.24 & 0.16 & 0.12 & 0.10 & 0.053 & 0.028 & 0.014 & 0.007 & 0.005 & .0214 & 0.0081 & 0.0027 & 0.0009 & 0.0006 \\
\hline \pm boost & 0.01 & 0.01 & 0.02 & 0.02 & 0.05 & 0.002 & 0.002 & 0.004 & 0.005 & 0.006 & .0012 & 0.0005 & 0.0008 & 0.0009 & 0.0012 \\
\hline \multicolumn{16}{|c|}{ Systematic Uncertainties } \\
\hline Continuum & 0.18 & 0.11 & 0.08 & 0.07 & 0.07 & 0.030 & 0.016 & 0.008 & 0.004 & 0.002 & $\overline{0101}$ & 0.0040 & 0.0012 & 0.0004 & 0.0004 \\
\hline Selection & 0.20 & 0.15 & 0.11 & 0.08 & 0.06 & 0.023 & 0.012 & 0.006 & 0.003 & 0.001 & 0114 & 0.0039 & 0.0014 & 0.0005 & 0.0001 \\
\hline$\pi^{0} / \eta$ & 0.07 & 0.05 & 0.04 & 0.02 & 0.01 & 0.012 & 0.006 & 0.003 & 0.002 & 0.001 & .0075 & 0.0023 & 0.0007 & 0.0003 & 0.0001 \\
\hline Other $B$ & 0.24 & 0.13 & 0.06 & 0.02 & 0.01 & 0.033 & 0.016 & 0.007 & 0.002 & 0.000 & 0.0124 & 0.0051 & 0.0017 & 0.0004 & 0.0000 \\
\hline Beam & 0.02 & 0.02 & 0.01 & 0.01 & 0.01 & 0.001 & 0.001 & 0.000 & 0.000 & 0.000 & 0.0006 & 0.0003 & 0.0001 & 0.0000 & 0.0000 \\
\hline resolution & 0.01 & 0.01 & 0.02 & 0.02 & 0.03 & 0.006 & 0.005 & 0.005 & 04 & 0. & 009 & 0.0006 & 0.0005 & 0.0004 & 0.0004 \\
\hline Unfolding & 0.01 & 0.00 & 0.00 & 0.01 & 0.01 & 0.002 & 0.001 & 0.001 & 0.001 & 0.002 & 0014 & 0.0008 & 0.0006 & 0.0003 & 0.0001 \\
\hline Model & 0.03 & 0.02 & 0.01 & 0.00 & 0.00 & 0.005 & 0.003 & 0.002 & 0.001 & 0.000 & 0.0014 & 0.0006 & 0.0002 & 0.0000 & 0.0000 \\
\hline$\gamma$ Detection & 0.03 & 0.02 & 0.01 & 0.00 & 0.00 & 0.005 & 0.003 & 0.002 & 0.001 & 0.000 & 0.0014 & 0.0006 & 0.0002 & 0.0000 & 0.0000 \\
\hline$B \rightarrow X_{d} \gamma$ & 0.01 & 0.01 & 0.01 & 0.01 & 0.01 & 0.000 & 0.000 & 0.000 & 0.000 & 0.000 & 0.0001 & 0.0000 & 0.0000 & 0.0000 & 0.0000 \\
\hline \multicolumn{16}{|c|}{ Correlation coefficients (combined statistical and systematic) } \\
\hline & \multicolumn{5}{|c|}{$\Delta \mathcal{B}$} & \multicolumn{5}{|c|}{$\left\langle E_{\gamma}\right\rangle$} & \multicolumn{5}{|c|}{$\Delta E_{\gamma}^{2}$} \\
\hline & 1.7 & 1.8 & 1.9 & 2.0 & 2.1 & 1.7 & 1.8 & 1.9 & 2.0 & 2.1 & 1.7 & 1.8 & 1.9 & 2.0 & 2.1 \\
\hline 1.7 & 1.000 & 0.959 & $\overline{0.811}$ & 0.699 & 0.604 & 0.455 & 0.322 & 0.114 & -0.083 & -0.142 & 0.848 & 0.857 & 0.722 & 0.528 & 0.445 \\
\hline 1.8 & & 1.000 & 0.942 & 0.839 & 0.720 & 0.269 & 0.129 & -0.073 & -0.251 & -0.291 & 0.807 & 0.878 & 0.822 & 0.678 & 0.568 \\
\hline$\Delta \mathcal{B} 1.9$ & & & 1.000 & 0.939 & 0.823 & 0.031 & -0.107 & -0.291 & -0.442 & -0.464 & 0.680 & 0.817 & 0.869 & 0.814 & 0.700 \\
\hline 2.0 & & & & 1.000 & 0.959 & -0.004 & -0.143 & -0.332 & -0.494 & -0.531 & 0.612 & 0.767 & 0.863 & 0.870 & 0.846 \\
\hline 2.1 & & & & & 1.000 & 0.023 & -0.107 & -0.296 & -0.476 & -0.548 & 0.546 & 0.689 & 0.795 & 0.848 & 0.910 \\
\hline 1.7 & & & & & & 1.000 & 0.967 & 0.838 & 0.636 & 0.489 & 0.342 & 0.149 & -0.094 & -0.252 & -0.174 \\
\hline 1.8 & & & & & & & 1.000 & 0.946 & 0.793 & 0.645 & 0.155 & -0.047 & -0.290 & -0.431 & -0.329 \\
\hline$\left\langle E_{\gamma}\right\rangle 1.9$ & & & & & & & & 1.000 & 0.942 & 0.824 & -0.066 & -0.280 & -0.516 & -0.640 & -0.530 \\
\hline 2.0 & & & & & & & & & 1.000 & 0.954 & -0.230 & -0.438 & -0.660 & -0.779 & -0.696 \\
\hline 2.1 & & & & & & & & & & 1.000 & -0.252 & -0.438 & -0.642 & -0.777 & -0.751 \\
\hline 1.7 & & & & & & & & & & & 1.000 & 0.945 & 0.782 & 0.581 & 0.497 \\
\hline 1.8 & & & & & & & & & & & & 1.000 & 0.935 & 0.782 & 0.677 \\
\hline$\Delta E_{\gamma}^{2} 1.9$ & & & & & & & & & & & & & 1.000 & 0.946 & 0.840 \\
\hline 2.0 & & & & & & & & & & & & & & 1.000 & 0.942 \\
\hline 2.1 & & & & & & & & & & & & & & & 1.000 \\
\hline
\end{tabular}

energy range $1.7 \mathrm{GeV} \leq E_{\gamma}^{\text {c.m.s }} \leq 2.8 \mathrm{GeV}$ in a fully inclusive way. For the first time $97 \%$ of the spectrum is measured [31] allowing the theoretical uncertainties to be reduced to a very low level. Using $605 \mathrm{fb}^{-1}$ of data taken at the $\Upsilon(4 S)$ and $68 \mathrm{fb}^{-1}$ taken below the resonance, we obtain $\mathcal{B}\left(B \rightarrow X_{s} \gamma: E_{\gamma}^{B}>1.7 \mathrm{GeV}\right)=$ $(3.31 \pm 0.19 \pm 0.37 \pm 0.01) \times 10^{-4}$, where the errors are statistical, systematic and due to the boost correction, respectively. This result is in agreement with the latest theoretical calculations [1, 2, 27]. The results can be used to place constraints on new physics [32] and determine SM parameters such as the $b$-quark mass [33].

\section{Acknowledgments}

We thank the KEKB group for excellent operation of the accelerator, the KEK cryogenics group for effi- cient solenoid operations, and the KEK computer group and the NII for valuable computing and SINET3 network support. We acknowledge support from MEXT and JSPS (Japan); ARC, DEST and A.J. Slocum (Australia); NSFC (China); DST (India); MOEHRD, KOSEF and KRF (Korea); KBN (Poland); MES and RFAAE (Russia); ARRS (Slovenia); SNSF (Switzerland); NSC and MOE (Taiwan); and DOE (USA). 


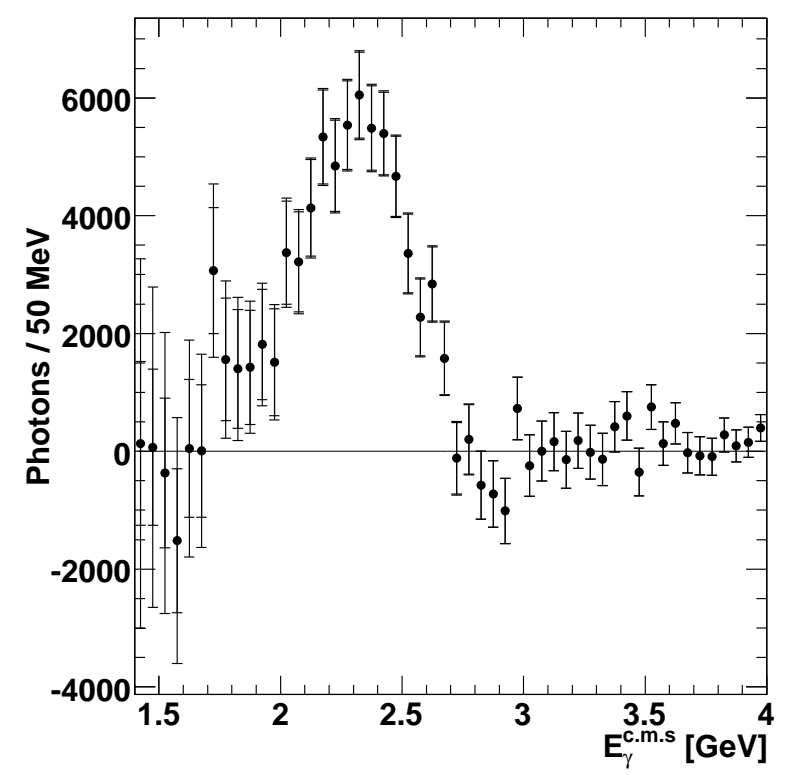

FIG. 4: The extracted photon energy spectrum of $B \rightarrow X_{s, d} \gamma$. The two error bars show the statistical and total errors.

[1] M. Misiak et al., Phys. Rev. Lett. 98, 022002 (2007), hep-ph/0609232.

[2] T. Becher and M. Neubert, Phys. Rev. Lett. 98, 022003 (2007), hep-ph/0610067.

[3] W. M. Yao et al. (Particle Data Group), J. Phys. G33, 1 (2006).

[4] S. Bertolini, F. Borzumati, A. Masiero, and G. Ridolfi, Nucl. Phys. B353, 591 (1991).

[5] P. L. Cho and M. Misiak, Phys. Rev. D49, 5894 (1994), hep-ph/9310332.

[6] K. Fujikawa and A. Yamada, Phys. Rev. D49, 5890 (1994).

[7] E. Barberio et al. (Heavy Flavor Averaging Group (HFAG)) (2007), arXiv:0704.3575 [hep-ex].

[8] I. Bigi and N. Uraltsev, Int. J. Mod. Phys. A17, 4709 (2002), hep-ph/0202175.

[9] K. Abe et al. (Belle), Phys. Lett. B511, 151 (2001), hepex/0103042.

[10] P. Koppenburg et al. (Belle) (2004), hep-ex/0403004.

[11] S. Chen et al. (CLEO), Phys. Rev. Lett. 87, 251807 (2001), hep-ex/0108032.

[12] B. Aubert et al. (BABAR), Phys. Rev. D72, 052004 (2005), hep-ex/0508004.

[13] B. Aubert et al. (BaBar), Phys. Rev. Lett. 97, 171803 (2006), hep-ex/0607071.

[14] B. Aubert et al. (BABAR), Phys. Rev. D77, 051103(R) (2008), arXiv:0711.4889 [hep-ex].

[15] S. Kurokawa and E. Kikutani, Nucl. Instrum. Meth. A499, 1 (2003), and other papers included in this Volume.

[16] A. Abashian et al., Nucl. Instrum. Meth. A479, 117 (2002).
[17] G. C. Fox and S. Wolfram, Phys. Rev. Lett. 41, 1581 (1978).

[18] R. Brun, R. Hagelberg, M. Hansroul, and J. C. Lassalle (1978), cERN-DD-78-2-REV.

[19] J. R. Andersen and E. Gardi, JHEP 06, 030 (2005), hep$\mathrm{ph} / 0502159$.

[20] B. Casey, Ph.D. thesis, University of Hawaii (2001), http://www.phys.hawaii.edu/ casey/thesis/.

[21] E. Barberio and Z. Was, Comput. Phys. Commun. 79, 291 (1994).

[22] D. J. Lange, Nucl. Instrum. Meth. A462, 152 (2001).

[23] A. Hocker and V. Kartvelishvili, Nucl. Instrum. Meth. A372, 469 (1996), hep-ph/9509307.

[24] A. L. Kagan and M. Neubert, Eur. Phys. J. C7, 5 (1999), hep-ph/9805303.

[25] B. O. Lange, M. Neubert, and G. Paz, JHEP 10, 084 (2005), hep-ph/0508178.

[26] B. O. Lange, M. Neubert, and G. Paz, Phys. Rev. D72, 073006 (2005), hep-ph/0504071.

[27] J. R. Andersen and E. Gardi, JHEP 01, 029 (2007), hep$\mathrm{ph} / 0609250$.

[28] D. Benson, I. I. Bigi, and N. Uraltsev, Nucl. Phys. B710, 371 (2005), hep-ph/0410080.

[29] P. Gambino and P. Giordano (2008), work in progress.

[30] T. Hurth, E. Lunghi, and W. Porod, Nucl. Phys. B704, 56 (2005), hep-ph/0312260.

[31] O. Buchmuller and H. Flacher, Phys. Rev. D73, 073008 (2006), hep-ph/0507253.

[32] O. Buchmueller et al., Phys. Lett. B657, 87 (2007), 0707.3447.

[33] C. Schwanda, P. Urquijo, E. Barberio, A. Limosani, et al. (Belle), submitted to Phys. Rev. D. (2008), 

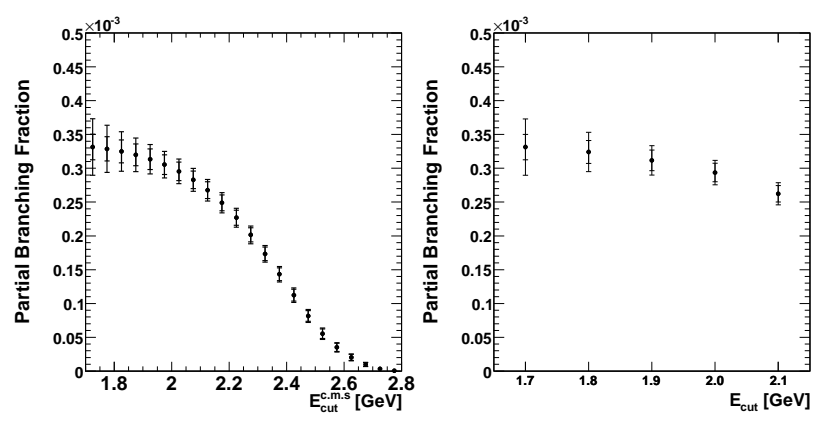

arXiv:0803.2158 [hep-ex].

[34] In the optimisation step the choice of signal model has a negligible effect on the measure of optimisation, suffice to say the choice of signal model should not be construed as preferential.
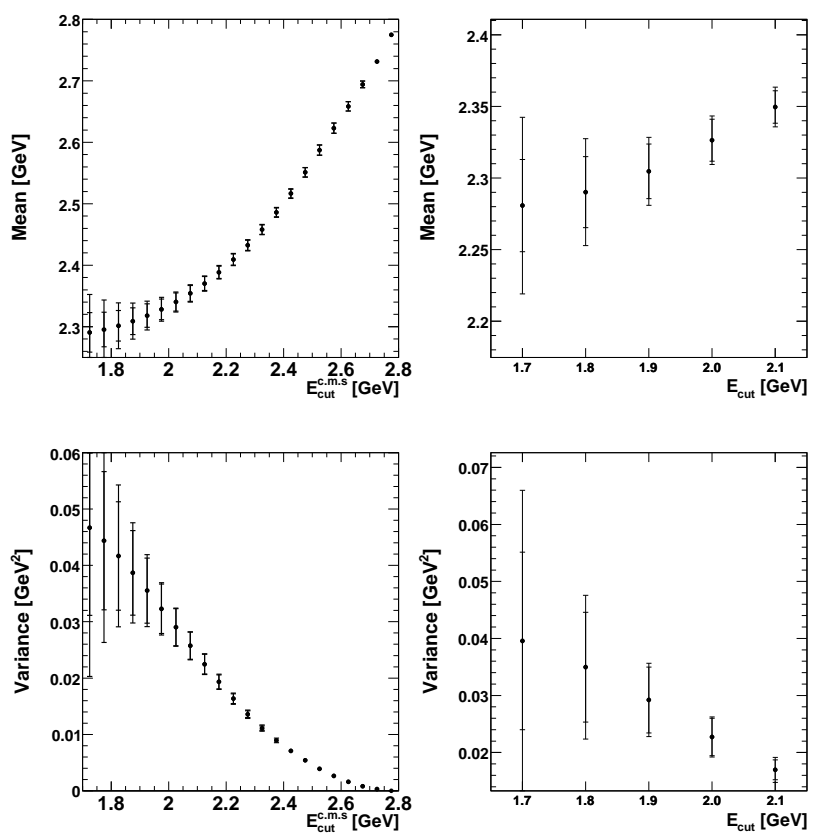

FIG. 5: (TOP) Partial branching fractions, (MIDDLE) mean, and (BOTTOM) variance of $B \rightarrow X_{s} \gamma$ in the (LEFT) c.m.s and (RIGHT) and in the rest frame of the $B$-meson for lower energy thresholds. The two error bars show the statistical and total errors. 